\title{
Molecular Cloning and Characterization of a Novel Calcium-dependent Protein Kinase Gene IiCPK2 Responsive to Polyploidy from Tetraploid Isatis indigotica
}

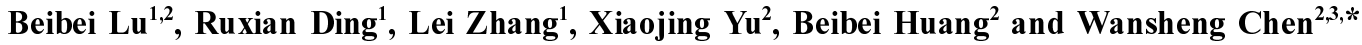 \\ ${ }^{1}$ Department of pharmacognosy, School of Pharmacy, Second Military Medical University, Shanghai 200433, China \\ ${ }^{2}$ Department of Pharmacy, Changzheng Hospital, Second Military Medical University, Shanghai 200003, China \\ ${ }^{3}$ Modern Research Center for Traditional Chinese Medicine, Second Military Medical University, Shanghai 200433, China
}

Received 29 March 2006, Accepted 7 June 2006

\begin{abstract}
A novel calcium-dependent protein kinase gene (designated as IiCPK2) was cloned from tetraploid Isatis indigotica. The full-length cDNA of $\mathrm{IiCPK} 2$ was $2585 \mathrm{bp}$ long with an open reading frame (ORF) of $1878 \mathrm{bp}$ encoding a polypeptide of 625 amino acid residues. The predicted IiCPK2 polypeptide included three domains: a kinase domain, a junction domain (or autoinhibitory region), and a C-terminal calmodulin-like domain (or calcium-binding domain), which presented a typical structure of plant CDPKs. Further analysis of IiCPK2 genomic DNA revealed that it contained 7 exons, 6 introns and the length of most exons was highly conserved. Semi-quantitative RTPCR revealed that the expression of $I i C P K 2$ in root, stem and leaf were much higher in tetraploid sample than that in diploid progenitor. Further expression analysis revealed that gibberellin $\left(\mathrm{GA}_{3}\right), \mathrm{NaCl}$ and cold treatments could upregulate the IiCPK2 transcription. All our findings suggest that IiCPK2 might participate in the cold, high salinity and $\mathrm{GA}_{3}$ responsive pathways.
\end{abstract}

Keywords: Defense/stress, Isatis indigotica, IiCPK2, Polyploidy, RACE

Database Accession No: DQ458915

Abbreviations: CPK: calcium-dependent protein kinase, CTAB: Cetyl trimethyl ammonium bromide, ORF: open reading frame, PCR: Polymerase chain reaction, RACE: rapid amplification of cDNA ends, RT-PCR: reverse transcriptase-polymerase chain reaction.

* To whom correspondence should be addressed.

Tel: 86-21-25070395; Fax: 86-21-25074575

E-mail: chenwanshengsmmu@gmail.com or chenws@vnet.citiz.net

\section{Introduction}

Calcium is a ubiquitous signal molecule and changes in cytosolic $\mathrm{Ca}^{2+}$ concentration are involved in plant responses to various stimuli, including light, environmental stresses, pathogen attack, and hormones (Poovaiah and Reddy, 1993; Bush, 1995). Calcium acts through various intracellular mediators, including calmodulin $(\mathrm{CaM})$ and $\mathrm{Ca}^{2+}$-regulated kinases (Sopory and Munshi, 1998). Plants contain a novel group of kinases, designated as $\mathrm{Ca}^{2+}$-dependent protein kinases (CDPKs; EC 2.7.1.37), which are found only in plants, green algae, and protozoa (Hrabak, 2003). So far, CDPKs have been shown to share a similar primary structure consisting of three domains: a kinase domain, a junction domain (or autoinhibitory region), and a C-terminal calmodulin-like domain (or calciumbinding domain). The kinase domain contains all 11 of the highly conserved subdomains of typical eukaryotic Ser/Thr protein kinases (Hanks et al., 1988). The autoinhibitory region contains a pseudo-substrate site that, in the absence of $\mathrm{Ca}^{2+}$, binds to the catalytic center, resulting in an inactive state of the kinase. However, the binding of $\mathrm{Ca}^{2+}$ to the calmodulinlike domain can induce a conformational shift for the release the pseudo-substrate domain from the active site and kinase activation (Harper et al., 1991; Harmon et al., 1994; Harmon et al., 2000). Besides these three conservative domains, all CDPKs have an N-terminal variable region, which often contains N-terminal acylation (including myristoylation and palmitoylation) sites for the subcellular localizations of CDPKs (Martin and Busconi, 2000; Rutschmann et al., 2002; Dammann et al., 2003).

The first isolation of a cDNA encoding a CDPK was from soybean (Harper et al., 1991). Since then, genes encoding CDPKs from a diverse spectrum of plants including Arabidopsis, maize, rice, and mung bean have been cloned and, in all cases, they are encoded by a multigene family (Kawasaki et al., 1993; Estruch et al., 1994; Urao et al., 1994; Breviario et al., 1995; Botella et al., 1996; Hong et al., 1996; 
Hrabak et al., 1996). This large number of genes suggests that individual isoforms have different functions and participate in multiple, distinct signaling pathways (Romeis et al., 2001). Increasing evidence shows that CDPKs are involved in environmental stress and hormone signaling. Drought, salt, cold, light, and hormones can influence the expression or activity of various CDPKs in plants (Sopory and Munshi, 1998). Increased CDPK activity and $\mathrm{Ca}^{2+}$-dependent protein phosphorylation were observed in rice seedlings exposed to cold (Li and Komatsu, 2000; Komatsu et al., 2001) and hormone treatment (Kawasaki et al., 1993; Karibe and Komatsu, 1997; Yang and Komatsu, 2000).

Polyploidy is recognized as a common phenomenon in the evolution of plants. It is estimated that 50 to $80 \%$ of angiosperms are polyploids, including many of our most important crop plants with the hallmarks of autopolyploidy (e.g. alfalfa and potato) or allopolyploidy (e.g. wheat, oat, cotton, coffee and canola) (Wendel, 2000). Polyploids often show novel phenotypes that are not present in their diploid progenitors or exceed the range of the contributing species (Levin, 1983; Ramsey and Schemske, 2002). Some of these traits, such as increased drought tolerance, apomixis (asexual seed production), pest resistance, flowering time, organ size and biomass, could allow polyploids to enter new niches or enhance their chances of being selected for use in agriculture. Many insights have emerged from recent explorations using laboratory generated or synthetic polyploids (Madlung et al., 2002; Han et al., 2003; Liu and Wendel, 2003; Pires et al., 2004; Adams and Wendel, 2005). Analysis of the most recent genome duplication event in Arabidopsis revealed that some classes of genes, such as those involved in transcription and signal transduction, had been preferentially retained and their transcription profiles were variant. (Blanc and Wolfe, 2004).

Isatis indigotica Fort., the same family Cruciferae with Arabidopsis thaliana, is a prevalent Chinese medicinal herb. After selection for five years, tetraploid I. indigotica $(2 \mathrm{n}=28)$ with better yield and enhanced resistance had been obtained, compared with diploid progenitor $(2 \mathrm{n}=14$ ) (Qiao et al., 1989). Whether the polyploidisation has effect on genes involved in signal transduction in I. indigotica is unknown. In the present study, a CDPK gene from tetraploid I. indigotica (IiCPK2) was cloned and characterized. The expression profiling analyses of IiCPK2 under stress conditions and phytohormone treatment suggest that $I i C P K$ may be involved in environmental stress and hormone signaling. Higher expression level and changed transcriptional profile in tetraploid I. indigotica compared with diploid progenitor suggests that $I i C P K$ probably participates in polyploidy evolution of I. indigotica.

\section{Materials and Methods}

Plant materials. Seeds of tetraploid and diploid I. indigotica were kindly provided by professor C.Z. Qiao of School of Pharmacy, Second Military Medical University, Shanghai, China. Seeds were pretreated with $75 \%$ alcohol for $1 \mathrm{~min}$, washed 3 times with distilled water, followed by the treatment of $0.1 \% \mathrm{HgCl}_{2}$ for $5 \mathrm{~min}$ and by 4 rinses with sterile distilled water. The sterilized seeds were then incubated between several layers of sterilized wet filter paper under $30^{\circ} \mathrm{C}$ for germination. Germinated seeds were sowed and cultured in small plastic flowerpots naturally. The seedlings were all grown at $25^{\circ} \mathrm{C}$ under $14 \mathrm{~h} \mathrm{light} / 10 \mathrm{~h}$ dark photoperiod cycles for two months until various treatments and RNA, DNA isolation.

Various treatments. The leaves from two-month-old tetraploid $I$. indigotica seedlings were sprayed with solution of $100 \mu \mathrm{M} \mathrm{GA}_{3}$, $100 \mu \mathrm{M}$ Abscisic Acid (ABA) and $250 \mathrm{mM} \mathrm{NaCl}$ respectively followed by RNA isolation. Another set of control plants were similarly treated with distilled water. For cold treatment, the seedlings were first grown at $28^{\circ} \mathrm{C}$ for $4 \mathrm{~d}$, transferred from $28^{\circ} \mathrm{C}$ to $4^{\circ} \mathrm{C}$ and grown for $8 \mathrm{~h}, 16 \mathrm{~h}$ and $32 \mathrm{~h}$ respectively, and then returned to $28^{\circ} \mathrm{C}$ for $32 \mathrm{~h}$.

RNA and DNA isolation. Total RNAs of different tissues including root, stem, leaf from two-month-old tetraploid and diploid I. indigotica were extracted using TRIzol Reagent (GIBCO BRL) according to the manufacturer's instruction (Jaakola et al., 2001) as well as total RNAs of tetraploid plant with various treatments. The genomic DNAs of tetraploid and diploid plant were isolated using a CTAB-based method (Rechards, 1995). The quality and concentration of RNA and DNA samples were examined by EB-stained agarose gel electrophoresis and spectrophotometer analysis.

Molecular cloning of the IiCPK2 Full-length cDNA. Molecular cloning of IiCPK2 from tetraploid I. indigotica was carried out by Rapid amplification of cDNA ends (RACE) method using a SMART $^{\mathrm{TM}}$ RACE cDNA Amplification Kit (Clontech).

For 3'RACE of $I i C P K 2$, about $100 \mathrm{ng}$ of total RNA was reverse transcribed with 3'-CDS primer (5'-AAGCAGTGGTATCAACGCA GAGTAC $(\mathrm{T})_{30} \mathrm{VN}-3^{\prime}, \mathrm{N}=\mathrm{A}, \mathrm{C}, \mathrm{G}$, or T; V = A, G, or C; Clontech USA) by BD PowerScript Reverse Transcriptase (Clontech). Universal Primer A Mix (UPM, 5'-CTAATACGACTCACTATAGGGCAAG CAGTGGTATCAACGCAGAGT-3', Clontech), Nested Universal Primer A (NUP, 5'-AAGCAGTGGTATCAACGCAGAGT-3', Clontech), gene-specific primers CPK3'GSP1 (5'-CCATTGTTGG GGTTGTAGAGGCTTGC-3', as 3' RACE first amplification primer) and CPK3'GSP2 (5'-GCATCGAGACCTCAAGCCCGAG AATT-3', as 3' RACE nest amplification primer) were used. The PCR was conducted in accordance with the protocol provided by the manufacture (Clontech). The nested amplified PCR product was purified and cloned into PMD18-T vector (TaKaRa) and then sequenced.

For 5' RACE of IiCPK2, about 100 ng of total RNA was reverse transcribed with 5'-CDS primer (5'-(T) $)_{25} \mathrm{VN}-3^{\prime}, \mathrm{N}=\mathrm{A}, \mathrm{C}, \mathrm{G}$, or T; V $=\mathrm{A}, \mathrm{G}$, or $\mathrm{C}$; Clontech USA) and SMART ${ }^{\mathrm{TM}}$ II A Oligonucleotide (5'-AAGCAGTGGTATCAACGCAGAGTACGCGGG-3', Clontech USA). Universal Primer A Mix (UPM), Nested Universal Primer A (NUP) (Clontech), gene-specific primers CPK5'GSP1 (5'-TCTGG AGCCACGCCGTCGATTTGTAC-3', as 5' RACE first amplification primer) and CPK5'GSP2 (5'-GCTACATAATACGGGCTACCAAC AAC-3', as 5' RACE nest amplification primer) were used. The PCR was conducted in accordance with the protocol provided by 
the manufacture (Clontech). The nested amplified PCR product was purified and cloned into PMD18-T vector (TaKaRa) and then sequenced. By aligning and assembling the products of $3^{\prime}$ and $5^{\prime}$ RACE, the full-length IiCPK2 from tetraploid I. indigotica was deduced and subsequently amplified by proof-reading PCR amplification with primers CPKF-S (5'-ATTAGACTGTTTTGAGC GTTTTGTGA-3') and CPKF-AS (5'-ACTCTTGTCTTTGGCTTTA GTTTTGTA-3'). The PCR procedure was conducted under the following conditions: $5 \mathrm{~min}$ at $94^{\circ} \mathrm{C}, 5$ cycles $\left(30 \mathrm{~s}\right.$ at $94^{\circ} \mathrm{C}, 30 \mathrm{~s}$ at $70^{\circ} \mathrm{C}, 3 \mathrm{~min}$ at $\left.72^{\circ} \mathrm{C}\right), 30$ cycles $\left(30 \mathrm{~s}\right.$ at $94^{\circ} \mathrm{C}, 30 \mathrm{~s}$ at $58^{\circ} \mathrm{C}, 3 \mathrm{~min}$ at $72^{\circ} \mathrm{C}$ ) and $10 \mathrm{~min}$ at $72^{\circ} \mathrm{C}$. The amplified PCR product was purified and cloned into PMD18-T vector (TaKaRa) and then sequenced. To examine whether the sequence of IiCPK2 in tetraploid plant was the same as that in diploid plant, the full-length IiCPK2 from diploid plant was amplified by RT-PCR (One-step RT-PCR Kit TaKaRa) using primers CPKF-S and CPKF-AS mentioned above. Aliquot of $2 \mu \mathrm{g}$ total RNA $(1 \mu \mathrm{g} / \mu \mathrm{l})$ of diploid plant was reversely transcribed at $50^{\circ} \mathrm{C}$ for $30 \mathrm{~min}$ and denatured at $94^{\circ} \mathrm{C}$ for $2 \mathrm{~min}$, followed by 35 cycles of amplification $\left(94^{\circ} \mathrm{C}\right.$ for $35 \mathrm{~s}, 56^{\circ} \mathrm{C}$ for $35 \mathrm{~s}, 72^{\circ} \mathrm{C}$ for $3 \mathrm{~min}$ ). The amplified PCR product was purified and cloned into PMD18-T vector (TaKaRa) and then sequenced.

Amplification of genomic sequence corresponding to the IiCPK2 Full-length cDNA. In order to detect whether there exist introns within the IiCPK2, PCR amplification was carried out using the same reaction system as for the cloning of the full-length cDNA except that the template was substituted by $1.5 \mu \mathrm{g}$ of total genomic DNA of tetraploid plant and the extension time at $72^{\circ} \mathrm{C}$ in the amplification cycles was prolonged up to $3.5 \mathrm{~min}$.

Sequence analyses of IiCPK2. Sequence alignments, ORF translation and molecular mass calculation of the predicted protein were carried out on Vector NTI Suite 8. Genbank BLASTs were carried out on NCBI (http://www.ncbi.nlm.nih.gov/), while bioinformatic analysis of the predicted IiCPK2 protein was carried out on website of Expasy Molecular Biology Server (http://www. expasy.org). Homology-based structural modeling was performed by Swiss-Model, and WebLab Viewer Lite 4.0 was used to display 3-D structures.

Expression profile of $\mathrm{IiCPK} 2$ in different tissues and stress, phytohormone assays. Semi-quantitative RT-PCR was used to investigate the expression profiling of $I i C P K 2$ in different tissues of tetraploid and diploid I. indigotica and under various stresses and phytohormone treatments. A mock treatment was carried out with $\mathrm{H}_{2} \mathrm{O}$ as a control to exclude any other factors inducing IiCPK2 gene expression that might result from the spraying. Total RNA was extracted separately from different tissues (leaves, roots, and stems) and under different treatments. After establishing agreement between the OD values of the RNAs, aliquot of $1 \mu \mathrm{g}$ total RNA $(1 \mu \mathrm{g} / \mu \mathrm{l})$ was used as the template in one-step RT-PCR analysis using One-step RT-PCR Kit (TaKaRa) with CPK-RT-S (5'-GTTCC TCCATTCTGGGCTGAATCCGA-3') and CPK-RT-AS (5'-TCTG ACCGCTTTTGTCGGCATCTATC-3') as primers. The template was reversely transcribed at $50^{\circ} \mathrm{C}$ for $30 \mathrm{~min}$ and denatured at $94^{\circ} \mathrm{C}$ for $2 \mathrm{~min}$, followed by 35 cycles of amplification $\left(94^{\circ} \mathrm{C}\right.$ for $35 \mathrm{~s}$, $50^{\circ} \mathrm{C}$ for $35 \mathrm{~s}, 72^{\circ} \mathrm{C}$ for $2 \mathrm{~min}$ ). The RT-PCR reaction for the housekeeping gene (18S gene) using specific primers $18 \mathrm{SF}$ (5'-
ATGATAACTCGACGGATCGC-3') and 18SR (5'-CTTGGATGT GGTAGCCGTTT-3') was performed as described above as the control except for 20 cycles. The densities of the target bands were measured with a WEALTEC Dolphin-DOC ultraviolet analyzer (WEALTEC). Each sample was assayed in triplicate.

\section{Results and Discussion}

Molecular cloning of the IiCPK2 full-length cDNA. Using the RACE method, cDNA ends of $1233 \mathrm{bp}$ and $1496 \mathrm{bp}$ were amplified by 3' RACE and 5'RACE respectively. 3' and 5' ends were assembled with Vector NTI Suite 8.0 and the deduced full-length $I i C P K 2$ cDNA was subsequently amplified by proof-reading PCR amplification with primers mentioned above. The full-length cDNA of IiCPK2 was $2585 \mathrm{bp}$, consisting of a 495-bp $5^{\prime}$ untranslated region, a 212-bp 3' untranslated region, and a 1878-bp ORF encoding a 625amino-acid protein (Fig. 1). The sequence of IiCPK2 in tetraploid plant was the same as that in diploid plant.

Characterization of IiCPK2 protein. The predicted IiCPK2 protein had a calculated molecular mass of $69.84 \mathrm{kDa}$ and a theoretical pI at 5.43. Protein-protein BLAST showed that on the amino acid level IiCPK2 protein shared high homology with CDPKs from other plant species. As shown in Fig. 1, the predicted IiCPK2 polypeptide included a kinase domain, a junction domain, and a C-terminal calcium-binding domain, which presented a typical structure of plant CDPKs. Besides these three conservative domains, IiCPK2 had an N-terminal variable region. By using Plant-Specific Myristoylation Predictor in PlantsP database (Gribskov et al., 2001) (http://plantsp. genomics.purdue.edu/plantsp/html/myrist.html), putative Nmyristoylation site was found in IiCPK2 as shown in Fig. 1, which might be required for the subcellular localization of IiCPK2.

Through Clustal W/X, full-length Alignment result showed that IiCPK2 protein shared $90 \%$ identity to AtCPK1 from Arobidopsis, 80\% identity to AtCPK2 from Arobidopsis, 73\% identity to NtCPK3 from tobacco and $67 \%$ identity to OsCPK3 from rice respectively (Fig. 2A). In C-terminal calcium-binding domain, four conserved $\mathrm{Ca}^{2+}$-binding $\mathrm{EF}$ hands were identified by ScanProsite (http://au.expasy.org/ tools/scanprosite/) (Fig. 2A). As shown in Figure 2B, the kinase domain of IiCPK2 contained 11-signature protein kinase subdomains characteristic of the serine/threonine protein kinase family (Hanks et al., 1988).

Secondary structure analysis of IiCPK2 protein was performed by SOMPA (http://npsa-pbil.ibcp.fr/cgi-bin/npsa_automat.pl? page $=$ npsa_sopma.html). The result showed that IiCPK2 consisted of $258 \alpha$-helices, $45 \beta$-turns joined by 80 extended strands and 242 random coils (Fig. 3). The secondary structure of IiCPK2 had high similarity in kinase domain and $\mathrm{Ca}^{2+}$-binding domain, while the $\mathrm{N}$-terminal variable region was not (Fig. 3). Subsequently, molecular homologous modeling of kinase domain and $\mathrm{Ca}^{2+}$-binding domain of 


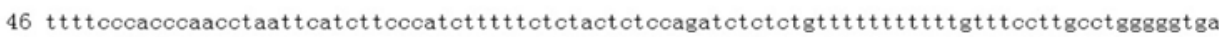

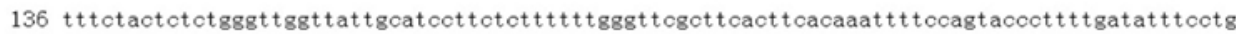

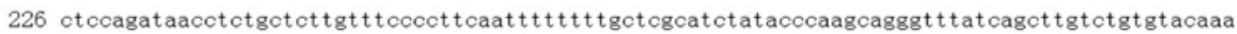

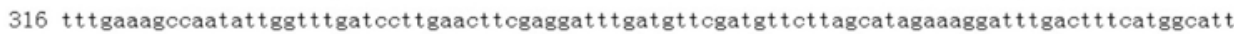
406 aggaatgagattagactgttttgagcgttttgtgatcagatct tagatgctatt tctggaaagagat aagacacgtt taggggtat tgg 496 atgggtaatacttgtgttggaccaagcagaaatgggttcttgcaatcogtttcggctgcaatgtggeggcetcgggatgcagatgattcg

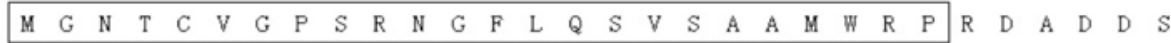
586 gtt tctcagagcaatggagat actgcaagtgaagetgtttcaggagagct togatctccatcatct gatcaggtccagaat aaaccccct

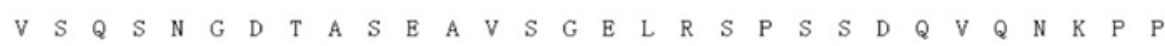

676 gagcagctcacaatgccaaagccagggactaacatcgagataaaggcgaaatccgatgtagagat tcagcctgaaatcaagctggaaacg

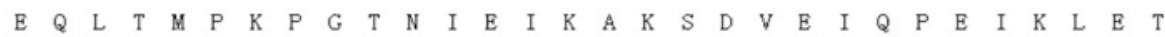

766 caagaggtgacaaaaccggagtccaagcoggagaccaagtcagagactaagcetgagaccaagtcggagactaagccggagaccaagtca $\begin{array}{lllllllllllllllllllllllllllllll}Q & E & V & T & K & P & E & S & K & P & E & T & K & S & E & T & K & P & E & T & K & S & E & T & K & P & E & T & K & S\end{array}$

856 gagactaagccggagacaaaagctgatactccggctaagcctaagaaacctaaacacatgaagagagtgtccagtgcaggtct taggact

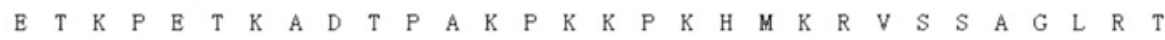

946 gagtcggtgttgcagaggaaaactgaaaact t taaggaat tctat tcct tgggaaggaaact tggacaagggcaatt tgggacgact t $t$

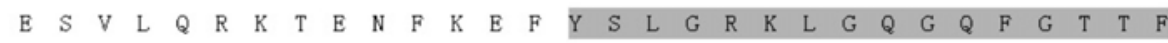

1036 ctatgcgtggagaagact tcagggaaggagtttgcctgcaagtctat tgctaagaggaagctgttgacogatgaggacgtggaagatgtg \begin{tabular}{lllllllllllllllllllllllllllllll}
\hline & $C$ & $V$ & $E$ & $K$ & $T$ & $S$ & $G$ & $K$ & $E$ & $F$ & $A$ & $C$ & $K$ & $S$ & $I$ & $A$ & $K$ & $R$ & $K$ & $L$ & $L$ & $T$ & $D$ & $E$ & $D$ & $V$ & $E$ & $D$ & $V$
\end{tabular}

1126 agaaggaaat tcagat tatgcatcact tggctggtcacccgaatgtcatatccat taaaggagct tatgaggatgt tctggeggtgcac $\begin{array}{lllllllllllllllllllllllllllllll}R & R & E & I & Q & I & M & H & H & L & A & G & H & P & N & V & I & S & I & K & G & A & Y & E & D & V & L & A & V & H\end{array}$

1216 ottgttatggagtgttgtgcaggtggcgagetttttgacagaat aat tcaacgeggtcactacacagagaggaaagetgct gagetcact $\begin{array}{llllllllllllllllllllllllllllllll}\mathrm{L} & \mathrm{V} & \mathrm{M} & \mathrm{E} & \mathrm{C} & \mathrm{C} & \mathrm{A} & \mathrm{G} & \mathrm{G} & \mathrm{E} & \mathrm{L} & \mathrm{F} & \mathrm{D} & \mathrm{R} & \mathrm{I} & \mathrm{I} & \mathrm{Q} & \mathrm{R} & \mathrm{G} & \mathrm{H} & \mathrm{Y} & \mathrm{T} & \mathrm{E} & \mathrm{R} & \mathrm{K} & \mathrm{A} & \mathrm{A} & \mathrm{E} & \mathrm{L} & \mathrm{T}\end{array}$

1306 agaaccatagtcggggtggtagaggct tgtcat tctct ggtgt tatgcatcgagacctcaagccogagaattt tctgtttgtcagtaaa $\begin{array}{llllllllllllllllllllllllllllllll}R & T & I & V & G & V & V & E & A & C & H & S & L & G & V & M & H & R & D & L & K & P & E & N & F & L & F & V & S & K\end{array}$

1396 cacgaagat tcactct tgaagacgat tgattttggactctctatgt tct taagccagatgatgtttttacagatgttgttggtagccog

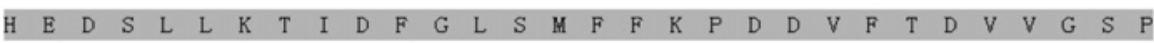

1486 tattatgtagctcoggaagt tct tcgaaagcat tatggtcotgaagctgatgtctggagtgctggagtgattgtatacattttgttaagt \begin{tabular}{lllllllllllllllllllllllllllllll}
\hline & $Y$ & V & A & P & E & V & L & $R$ & K & H & Y & G & P & E & A & D & V & W & S & A & G & V & I & V & Y & I & L & L & $S$
\end{tabular}

1576 ggagt tcctccattctgggctgaatcogaacaaggtattttcgaacaggtcotccatggtgatct tgact tt tcatcogatcoctggccg $\begin{array}{lllllllllllllllllllllllllllllllll}\text { G } & V & P & P & F & W & A & E & S & E & Q & G & I & F & E & Q & V & L & H & G & D & L & D & F & S & S & D & P & W & P\end{array}$

1666 agcatatctgaaagtgcaaaagact tagtgaggaaaatgct tgt tcgggatcccaagagaagactaactgcacaccaagtat tatgtcat $\begin{array}{lllllllllllllllllllllllllllllllll}S & I & S & E & S & A & K & D & L & V & R & K & M & L & V & R & D & P & K & R & R & L & T & A & H & Q & V & L & C & H\end{array}$

1756 ccatgggtacaaatcgacggegtggetccagacaaacctctagattctgetgttctgagcogtatgaagcaattttctgcaatgaacaas

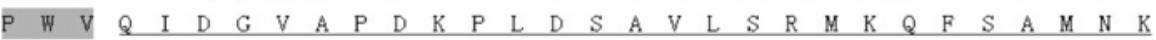

1846 t tcaagaaaatggetct tagagtcat tgctgagagct tatctgaagaggaaatagcogget tgaaagaaatgtt taatatgatagatgcc $\begin{array}{lllllllllllllllllllllllllllllllll}\mathrm{F} & \mathrm{K} & \mathrm{K} & \mathrm{M} & \mathrm{A} & \mathrm{L} & \mathrm{R} & \mathrm{V} & \mathrm{I} & \mathrm{A} & \mathrm{E} & \mathrm{S} & \mathrm{L} & \mathrm{S} & \mathrm{E} & \mathrm{E} & \mathrm{E} & \mathrm{I} & \mathrm{A} & \mathrm{G} & \mathrm{L} & \mathrm{K} & \mathrm{E} & \mathrm{M} & \mathrm{F} & \mathrm{N} & \mathrm{M} & \mathrm{I} & \mathrm{D} & \mathrm{A}\end{array}$ 1936 gacaaaagcggtcagataact t tcgaagaactgaaagcaggactaaaacgagtaggggcgaatctcaaagagtcagagat tctcgact tg \begin{tabular}{|llllllllllllllllllllllllllllllll}
\hline D & $K$ & S & $G$ & $Q$ & I & T & F & E & E & L & K & A & G & L & K & R & V & G & A & N & L & K & E & S & E & I & L & D & L \\
\hline
\end{tabular}

2026 atgcaagctgctgatgtggacaacagtggaacaatagactacaaagagt toatagcagcaacat tgcat t taaacaagatagagagagaa \begin{tabular}{llllllllllllllllllllllllllllllll}
\hline$M$ & $Q$ & $A$ & $A$ & $D$ & $V$ & $D$ & $N$ & $S$ & $G$ & $T$ & I & $D$ & Y & K & E & F & I & A & A & T & L & H & L & N & K & I & E & R & E \\
\hline
\end{tabular}

2116 gaccatt tgtttgcagcett tacatact t tgacaaagatgggagcggt tatatcaccocggacgaget tcaacaaget tgtgaggagt $t$ t \begin{tabular}{|lllllllllllllllllllllllllllllll}
\hline P & $H$ & L & F & A & A & F & T & Y & F & D & K & D & G & S & G & Y & I & T & P & D & E & L & $Q$ & $Q$ & A & C & E & E & F \\
\hline
\end{tabular}

2206 ggagttgaggatgtccgcatagaagaaatgatgcgtgatgttgatcaagacaatgacgggcgaatagattataacgagtttgtggcgatg

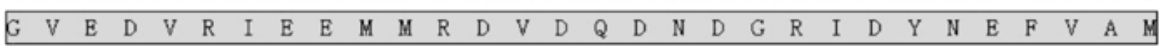

2296 atgcagaaagggagcatcacaggagttccagtgaagatgggactagagaaaagctttagcattgctct taaactctagtt tcaatct tt

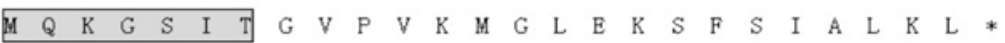

2386 taagt tacaaaactaaagccaaagacaagagt tcct tctgatgatatatgat tgtcgaagacaaa agcacagccaagat tctct t t tcg

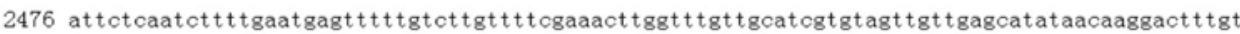
2566 caaaaaaaaaaaaaaaaa

Fig. 1. The full-length cDNA sequence and the deduced amino acid sequence of IiCPK2. The start codon (atg) is in bold and the stop codon (tag) is in italics. The putative $N$-myristoylation site $\left(\mathrm{A}_{1}-\mathrm{A}_{24}\right)$ is boxed with white background, the kinase domain $\left(\mathrm{A}_{165}-\mathrm{A}_{423}\right)$ is shown in the grey background, the junction domain $\left(\mathrm{A}_{424}-\mathrm{A}_{465}\right)$ is underlined. The calcium-binding domain $\left(\mathrm{A}_{466}-\mathrm{A}_{607}\right)$ is boxed with grey background. 


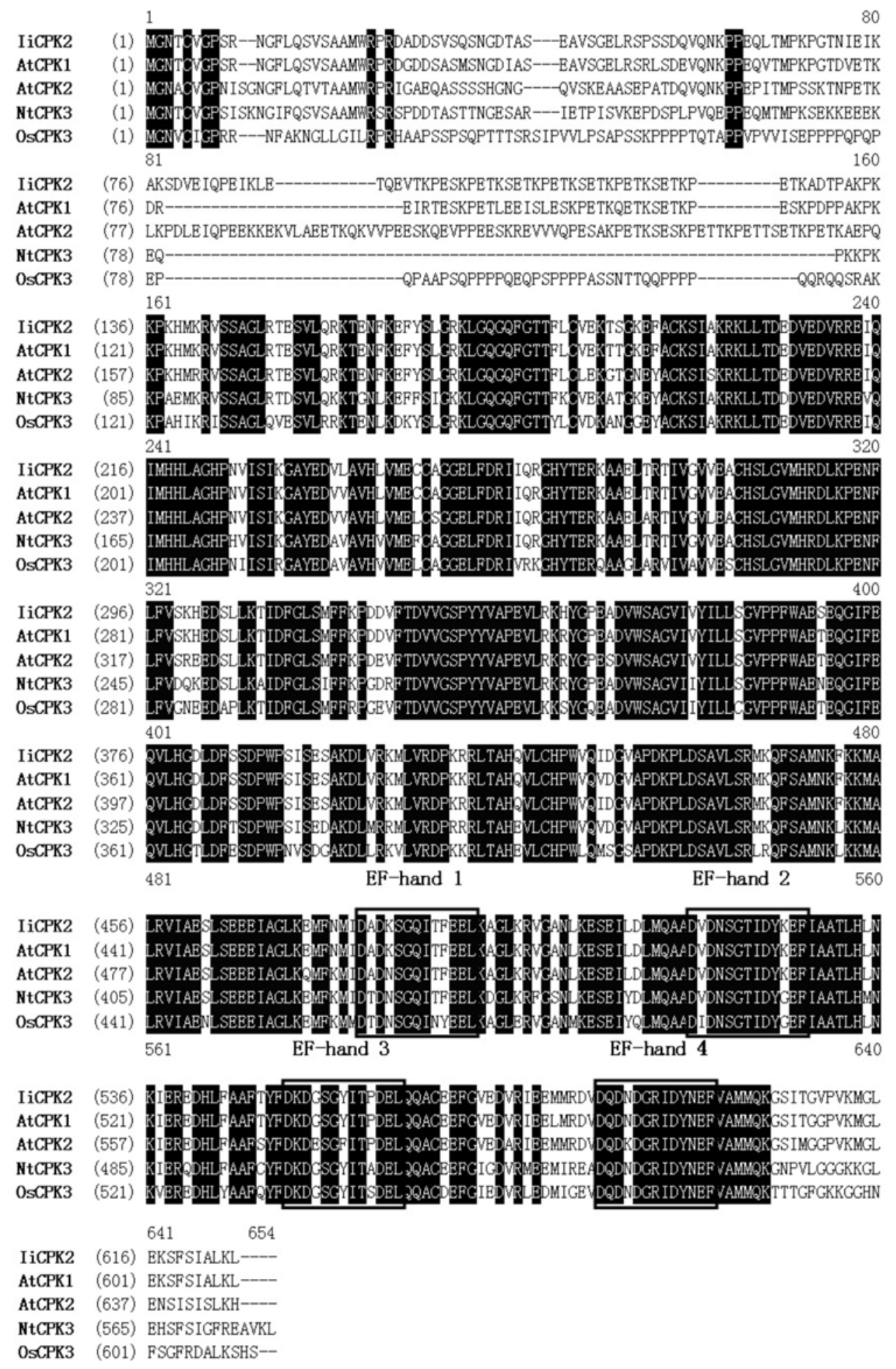

Fig. 2. The alignments of full-length of deduced IiCPK2 protein and kinase domain of IiCPK2. A: full-length alignment of IiCPK2. Identical amino acid residues are denoted by black backgrounds. The four EF-hands are shown in black boxes. B: Alignment of kinase domain of IiCPK2. Identical amino acid residues are denoted by black backgrounds. Roman numerals indicate the eleven canonical subdomains of protein kinases identified by Hanks (1988). AtCPK1 (A. thaliana, AT5G04870); AtCPK2 (A. thaliana, AAG51400); NtCPK3 (Nicotianan tobacum, CAC82999); OsCPK3 (Oryza sativa, ABA98539); MLCK-K (Oryctolagus cuniculus, P07313). 

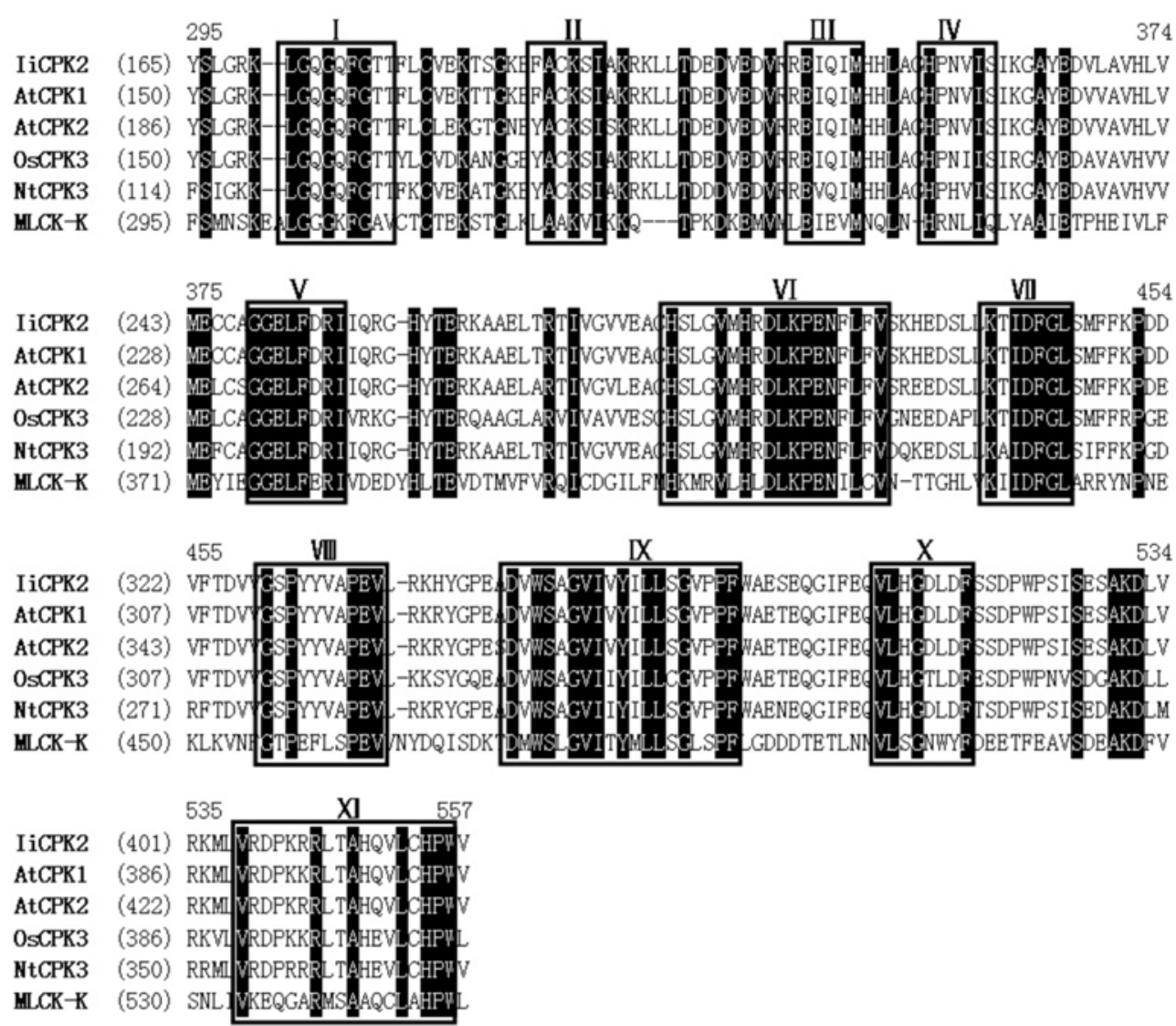

Fig. 2. Continued.

IiCPK2 was performed by Swiss Model Server (http:// swissmodel.expasy.org/) respectively. The result indicated that folding mode and spatial configuration of two domains of IiCPK2 shared high similarity with AtCPK1 from Arobidopsis (Fig. 4). Polypeptide of four EF-hands formed four loop structures in different directions which might be favourable for binding calcium (Fig. 4).
Genomic DNA analysis of $\mathrm{IiCPK2}$ gene. Genomic Sequence Corresponding to the IiCPK2 Full-length cDNA was $3638 \mathrm{bp}$ (GenBank Accession No: DQ458916). The alignment result of IiCPK2 cDNA sequence and the genomic sequence indicated that the IiCPK2 gene contained 7 exons and 6 introns (Fig. 5). The intron-exon junctions obeyed the GT/AG rule (Shapiro and Senapathy, 1987) (Fig. 5). Comparison of

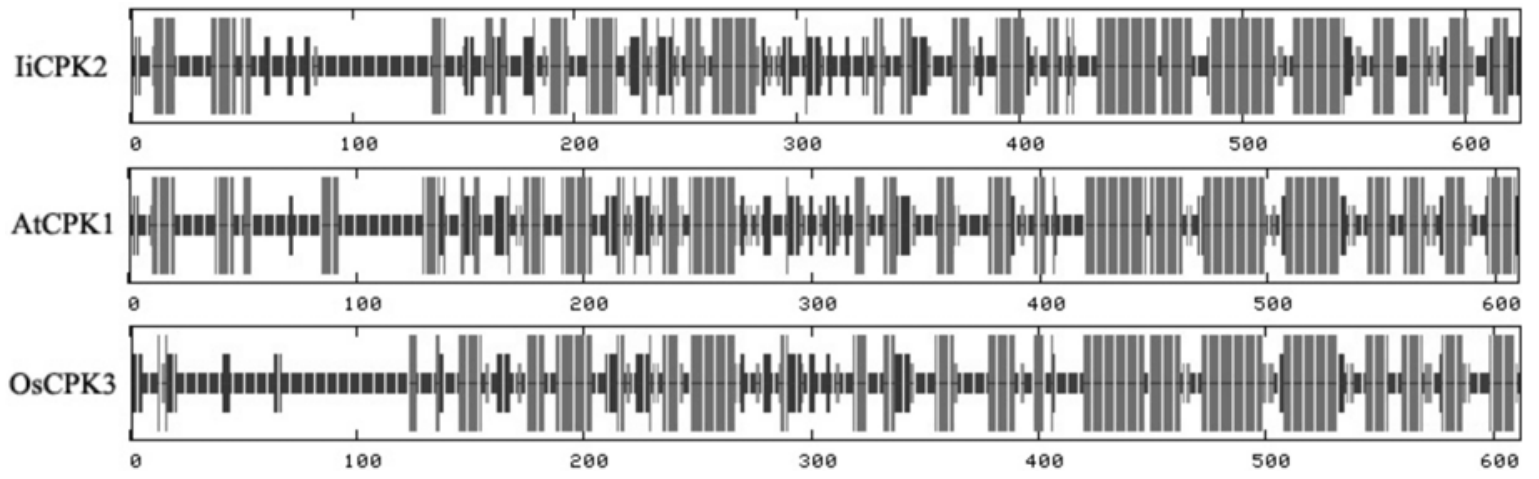

Fig. 3. The secondary structure of the deduced IiCPK 2 protein. AtCPK 1 (the highest identity); OsCPK 3 (the lowest identity). Alpha helix, extended strand, beta turn and random coil are denoted by the longest, the second longest, the second shortest and the shortest bars respectively. 


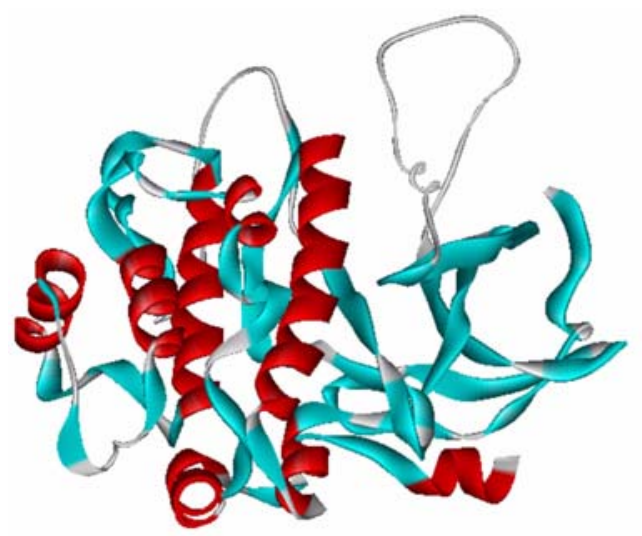

(A)

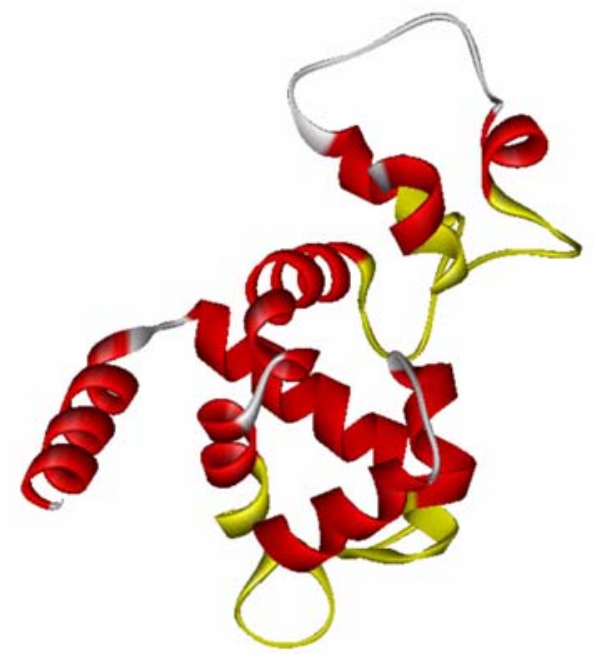

(C)

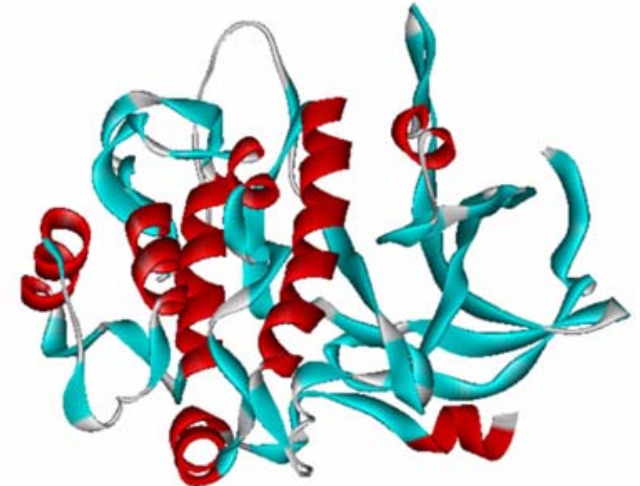

(B)

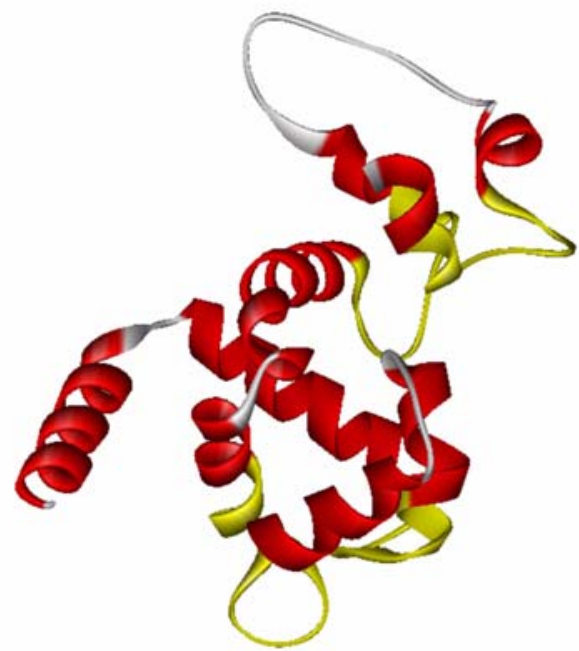

(D)

Fig. 4. Three-dimensional structures of the deduced IiCPK2 kinase domain and calcium-binding domain. A: kinase domain of IiCPK2, B:kinase domain of AtCPK1, C: calcium-binding domain of IiCPK2, D: calcium-binding domain of AtCPK1. $\alpha$-helices are indicated in red and $\beta$-sheets are indicated in blue. Turns and loops are indicated by gray lines. Four EF-hands in C and D are labeled by yellow.

the genomic sequence with the cDNA revealed that the $5^{\prime}$ portion of exon1 encoded the 5'-untranslated region (UTR); the protein-coding region extended from 3 ' portion of exon 1 through exon 7; exon7 encoded part of the IiCPK2 protein and the entire 3'-UTR. Further sequence analysis revealed that the length of exons of IiCPK2 was high conserved compared with genomic sequence from Arobidopsis ( $A t C P K 1$ and $A t C P K 2)$ and Rice (OsCPK3) except for exon 1 (Fig. 5), which differed somewhat in sequence and length. The probable reason was that this part of the protein contained Nterminal variable region and had a slightly different function in I. indigotica.

Effects of applied stress and phytohormones on IiCPK2 transcription. CDPKs were suggested to be associated with various stress tolerance and hormones in different plant species (Sopory and Munshi, 1998). To clarify the possible role of IiCPK2 in tetraploid I. indigotica, the expression of the
IiCPK2 in response to $\mathrm{NaCl}$, low-temperature, exogenous $\mathrm{ABA}$ and $\mathrm{GA}_{3}$ was analyzed by semi-quantitative RT-PCR. When treated with $250 \mathrm{mM} \mathrm{NaCl}$ and $100 \mu \mathrm{M} \mathrm{GA}$ by spraying, the expression of IiCPK2 was induced and reached the highest $16 \mathrm{~h}$ after the treatment, and then decreased gradually thereafter (Fig. 6). Under cold treatment, the expression of $I i C P K 2$ was up-regulated and reached the highest $16 \mathrm{~h}$ after the treatment. However, ABA treatment had no significant effect on the expression of IiCPK2 (Fig. 6).

The results verified that the expression of IiCPK2 might be relevant to the stress tolerance including low-temperature and salt tolerance. The relationship between low-temperature, salt and $\mathrm{GA}_{3}$ regulated gene expression was not understood, but accumulating evidence suggested that independent signaltransduction pathways may operate in response to different environmental signals (Nordin et al., 1991; Nordin et al., 1993; Yamaguchi-Shinozaki and Shinozaki, 1994; Zhu, 2002; Kim et al., 2003). Previous evidence suggested that CDPKs 


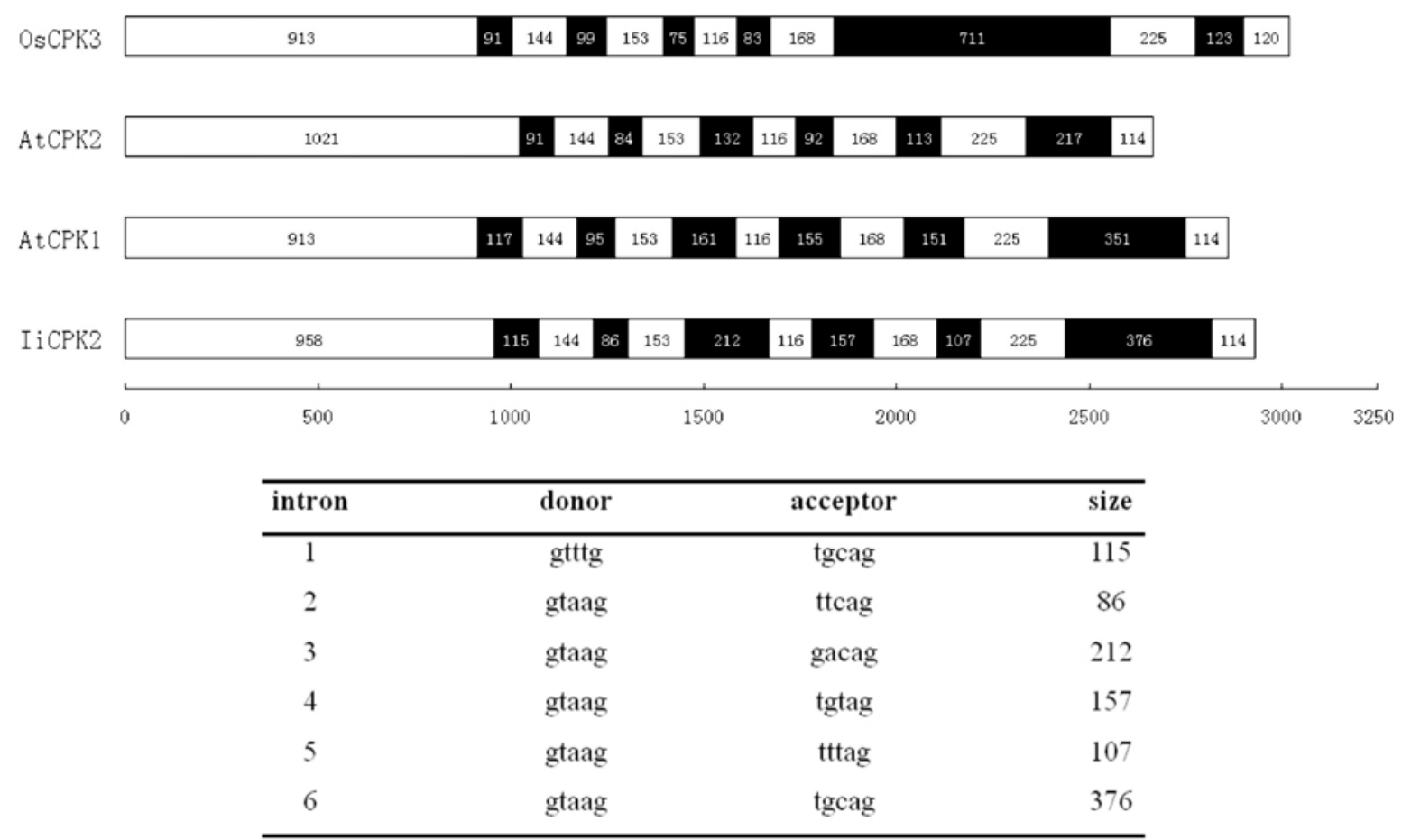

Fig. 5. Genomic organization of the $I i C P K 2$ and other CDPK genes (corresponding to coding region). In this schematic diagram, the exons are represented as boxes without background and the introns filled with grey background.

may be involved in certain plant hormone responses. For example, the activity of a rice seed membrane CDPK was increased by $\mathrm{GA}_{3}$ (Abo-El-Saad and $\mathrm{Wu}, 1995$ ). Furthermore, a $\mathrm{GA}_{3}$-induced increase in $\mathrm{Ca}^{2+}$ influx had been observed in barley aleurone protoplasts (Gilroy and Jones, 1992), providing a possible mechanism to link the $\mathrm{GA}_{3}$ response with activation of CDPKs. In this study, IiCDPK2 was upregulated in response to $\mathrm{GA}_{3}$ treatment (Fig. 6). The results suggest that IiCDPK2 might act in a $\mathrm{GA}_{3}$-mediated signaling.

Dehydration stresses including drought, low-temperature and high salinity adversely affect the growth of plants and the productivity of corps (Rabbani et al., 2003; Shen et al., 2004). Both ABA-independent and ABA-dependent signal transduction pathways link dehydration and gene expression (Guerrero et al., 1990; Yamaguchi-Shinozaki et al., 1992; YamaguchiShinozaki and Shinozaki 1993). Low-temperature inducible genes had been shown to be regulated by three separate signal pathways, one of which is ABA-independent (Nordin et al., 1991; Gilmour and Thomashow, 1991). In the present study, low-temperature and high salinity stress up-regulated the expression of $I i C P K 2$, while ABA had no significant effect on the expression of IiCPK2 (Fig. 6). These results suggest that $I i C P K 2$ gene induced expression should occur through ABAindependent pathway.

An alternative explanation for the involvement of IiCPK2 in cold stress, high salinity stress and GA signaling was crosstalk between these pathways. Signaling pathways have to be regarded as complex networks (Zhao et al., 2005). These signal networks are characterized by multiple points of convergence and divergence that enable integration of signaling pathways at different levels and provide the molecular basis for appropriate downstream responses. For example, Nicotiana benthamiana plants with reduced levels of NtCDPK show severe abnormalities in cell morphology, spontaneous necrotic lesions, and increased expression of marker genes for the plant defense response (Lee et al., 2003). These results suggest that plant cell development and defense responses could be interconnected at the level of NtCDPK1. Similarly, cold and high salinity stress responsiveness and $\mathrm{GA}_{3}$ dependent signal transduction may be promoted through distinct signaling pathways that crosstalk at the level of IiCPK2.

Divergence of transcription profiles of $I i C P K 2$ in different tissues between diploid and tetraploid $I$. indigotica. It was suggested that some classes of genes, such as those involved in transcription and signal transduction, had been preferentially retained and their transcription profiles were variant after genome duplication event in Arabidopsis. (Blanc and Wolfe, 2004). To determine whether the expression profile of IiCPK2 was influenced by genome duplication, total RNA was extracted from different tissues (roots, stems and leaves) of diploid and tetraploid I. indigotica and used for semiquantitative RT-PCR. It was found that the $I i C P K 2$ was constitutively expressed in all the tested tissues including root, stem and leaf in both tetraploid and diploid plant (Fig. 7). As expected, the expression level of IiCPK2 in root, stem and leaf from tetraploid I. indigotica was much higher than that of diploid samples (Fig. 7). Furthermore, the result was interesting 

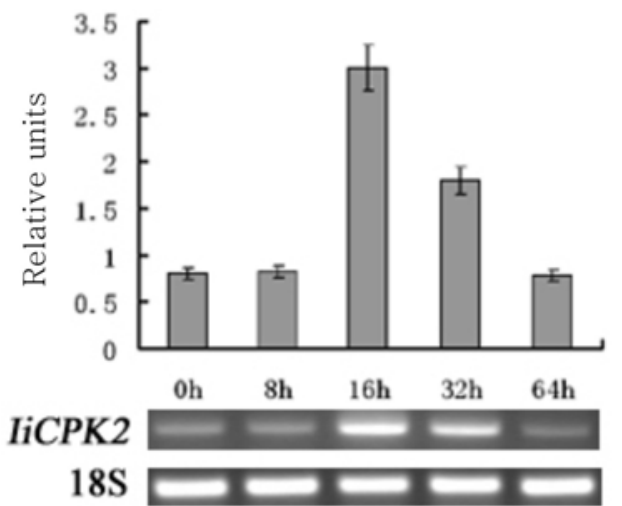

Cold

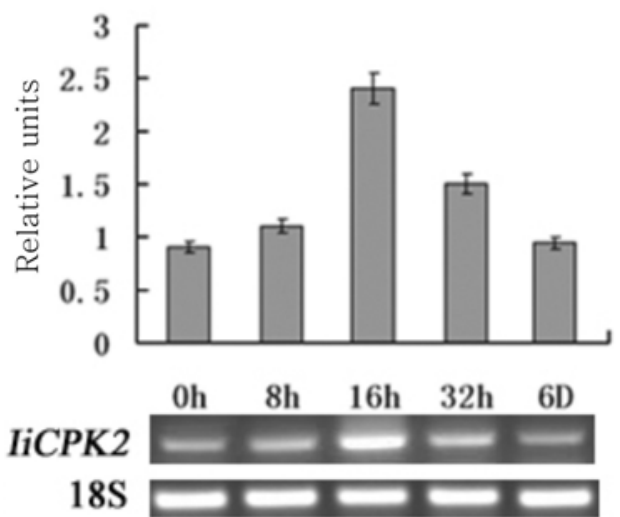

$\mathrm{GA} 3$

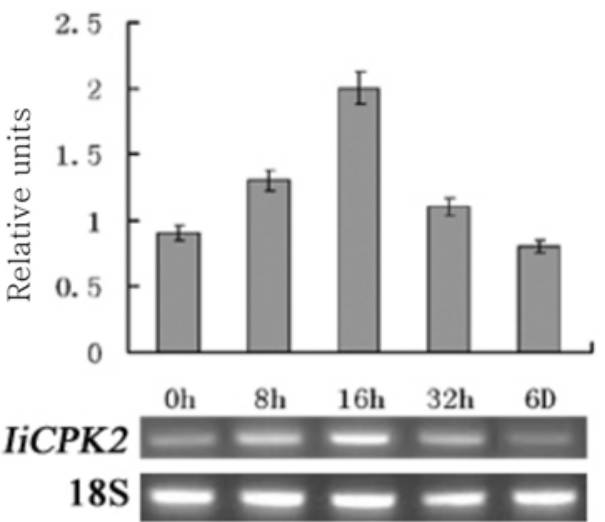

$\mathrm{NaCl}$

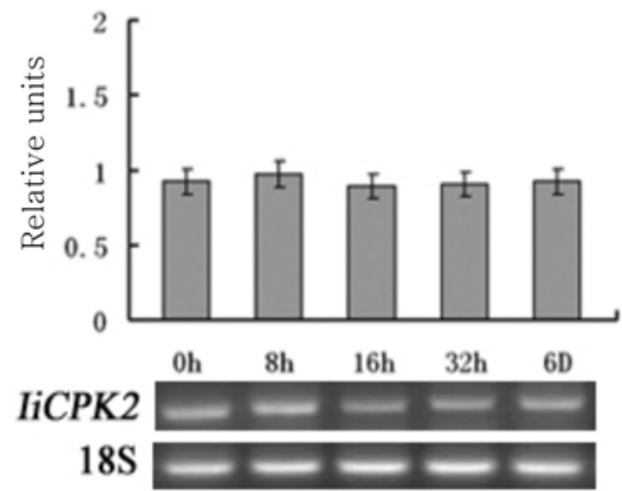

ABA

Fig. 6. Effects of applied stress and phytohormones on IiCPK2 transcript levels. Data represents the mean values $\pm \mathrm{SE}$ of three replicates.

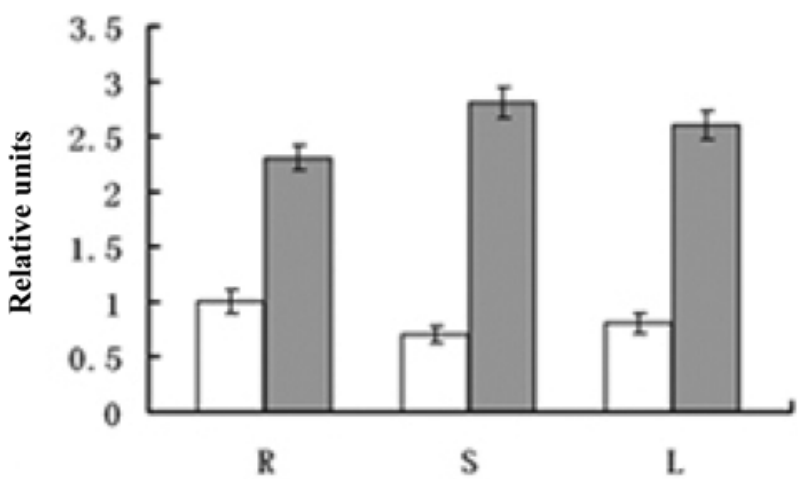

IICDPK2

$18 \mathrm{~S}$

Fig. 7. Expression profiling of $I i C P K 2$ in different tissues of tetraploid and diploid I. indigotica.R: root; S: stem; L: leaf. White columns indicate $I i C P K 2$ expression of diploid plant; Grey columns indicate IiCPK2 expression of tetraploid plant. Data represents the mean values $\pm \mathrm{SE}$ of three replicates.

that the highest expression of $I C P K 2$ in diploid plant was found in root, followed by in leaf, and stem, while the expression of IiCPK2 in tetraploid plant was almost the same in these tissues (Fig. 7). These results suggested that transcription profiles of $I i C P K 2$ might be influenced by genome duplication.

One of the intriguing findings is the level of IiCPK2 expression in the tetraploid I. indigotica is more than twice of that found in the diploid plant, especially in stems. The probable explanation for this phenomenon is altered regulatory networks in tetraploid I. indigotica after genome duplication. The expression of most genes is dependent on networks of regulators, such as transcription factors, that are organized into hierarchies (Guo and Birchler, 1994; Birchler et al., 2001). The numbers of regulators in diploid networks is high, but in polyploids they can be expanded several fold. The functioning of regulatory networks in polyploids will depend, in part, on how effectively regulators encoded by the contributing genomes interact. Polyploidy could alter the functioning of these networks by potentially increasing the number of different interacting regulators through an increase in number of alleles (Osborn et al., 2003). Similarly, regulatory network functioning might be severely altered in tetraploid I. indigotica derived from diploids. Therefore, the overall effects on the expression of genes in tetraploid I. indigotica at the ends of regulatory cascades might be significant.

In conclusion, we have cloned a new plant CDPK gene 
from tetraploid I. indigotica and named it IiCPK2. Although the details of $I i C P K 2$ function are still unclear, its sequence characterizations and induced expression responsive to $\mathrm{NaCl}$, low-temperature and $\mathrm{GA}_{3}$ suggest that IiCPK2 might participate in the cold, high salinity and hormone response pathways. Higher expression and changed transcription profiles of $I i C P K 2$ in tetraploid plant suggest that polyploidisation might have effect on $\mathrm{IiCPK} 2$ expression, which also might partially result in increased adaptable flexibility to environmental cold and high salinity conditions for tetraploid I. indigotica compared with diploid plants. In succession, the cloning of $I i C P K 2$ enables us to test whether the IiCPK2 gene could increase the tolerance and recovery from cold and high salinity stress of the whole transgenic plants by transferring it into model plants in the future.

Acknowledgments This research was financially supported by the National Natural Science Foundation of China (30371746).

\section{References}

Abo-El-Saad, M. and Wu, R. (1995) A rice membrane calciumdependent protein kinase is induced by gibberellin. Plant Physiol. 108, 787-793.

Adams, K. L. and Wendel, J. F. (2005) Polyploidy and genome evolution in plants. Curr. Opin. Plant Biol. 8, 135-141.

Birchler, J. A., Bhadra, U., Bhadra, M. P. and Auger, D. L. (2001) Dosage-dependent gene regulation in multicellular eukaryotes: Implications for dosage compensation, aneuploid syndromes, and quantitative traits. Dev. Biol. 234, 275-288.

Blanc, G. and Wolfe, K. H. (2004) Functional divergence of duplicated genes formed by polyploidy during Arabidopsis evolution. Plant Cell 16, 1679-1691.

Botella, J. R., Arteca, J. M., Somadevilla, M. and Arteca, R. N. (1996) Calcium-dependent protein kinase gene expression in response to physical and chemical stimuli in mungbean (Vigna radiata). Plant Mol. Biol. 30, 1129-1137.

Breviario, D., Morello, L. and Giani, S. (1995) Molecular cloning of two novel rice cDNA sequences encoding putative calciumdependent protein kinases. Plant Mol. Biol. 27, 953-967.

Bush, D. S. (1995) Calcium regulation in plant cell and its role in signaling. Annu. Rev. Plant Physiol. Plant Mol. Biol. 46, 95122.

Dammann, C., Ichida, A., Hong, B., Romanowsky, S. M., Hrabak, E. M., Harmon, A. C., Pickard, B. G. and Harper, J. F. (2003) Subcellular targeting of nine calcium-dependent protein kinase isoforms from Arabidopsis, Plant Physiol. 132, 1840-1848.

Estruch, J. J., Kadwell, W., Merlin, E. and Crossland, L. (1994) Cloning and characterization of a maize pollen-specific calcium-dependent calmodulin-independent protein kinase. Proc. Natl. Acad. Sci. USA 91, 8837-8841.

Gilmour, S. J. and Thomashow, M. F. (1991) Cold acclimation and cold-regulated gene expression in $\mathrm{ABA}$ mutants of Arabidopsis thaliana. Plant Mol. Biol. 17, 1233-1240.

Gilroy, S. and Jones, R. L. (1992) Giberellic acid and abscisic acid coordinately regulate cytoplasmic calcium and secretory activity in barley aleurone protoplasts. Proc. Natl. Acad. Sci. 89, 3591-3595.

Gribskov, M., Fana, F., Harper, J, Hope, D. A., Harmon, A. C., Smith, D. W., Tax, F. E. and Zhang, G. (2001) PlantsP: a functional genomics database for plant phosphorylation. Nucleic. Acids. Res. 29, 111-113.

Guerrero, F. D., Jones, J. T. and Mullet, J. E. (1990) Turgorresponsive gene transcription and RNA levels increase rapidly when pea shoots are wilted. Sequence and expression of three inducible genes. Plant Mol. Biol. 15, 11-26.

Guo, M. and Birchler, J. A. (1994) Trans-acting dosage effects on the expression of model gene systems in maize aneuploids. Science 266, 200-214.

Han, F. P., Fedak, G., Ouellet, T. and Liu, B. (2003) Rapid genomic changes in interspecific and intergeneric hybrids and allopolyploids of Triticeae. Genome 46, 716-723.

Hanks, S. K., Quinn, A. M. and Hunter, T. (1988) The protein kinase family conserved features and deduced phylogeny of the catalytic domain. Science $\mathbf{2 4 8}, \mathbf{4 2 - 5 2 .}$

Harmon, A. C., Yoo, B.C. and McCaffery, C. (1994) Pseudosubstrate inhibition of CDPK, a protein kinase with a calmodulin-like domain, Biochemistry 33, 7278-7287.

Harmon, A. C., Gribskov, M. and Harper, J. F (2000). CDPK's-A kinase for every Ca2+ signal? Trends Plant Biol. 5, 154-159.

Harper, J. F., Sussman, M. R., Schaller, G. E., Putnam-Evans, C., Charbonneau, H. and Harmon, A. C. (1991) A calciumdependent protein kinase with a regulatory domain similar to calmodulin. Science 252, 951-954.

Hong, Y., Takano, M., Liu, C. M., Gasch, A., Chye, M. L. and Chua, N-H. (1996) Expression of three members of the calcium-dependent protein kinase gene family in Arabidopsis thaliana. Plant Mol. Biol. 30, 1259-1275.

Hrabak, E. M., Dickmann, L. J., Satterlee, J. S. and Sussman, M. R. (1996) Characterization of eight new members of the calmodulin-like domain protein kinase gene family from Arabidopsis thaliana. Plant Mol. Biol. 31, 405-412.

Hrabak, E. M. (2003) Calcium-dependent protein kinase and their relatives; in Advances in Botanies Sciences, Plant Protein Kinase, Kreis, M. and Walker, J. C. (eds.) pp. 185-223, Academic Press, New York, USA.

Jaakola, L., Pirttila, A. M., Halonen, M. and Hohtola, A. (2001) Isolation of high quality RNA from bilberry (Vaccinium myrtillus L.) fruit. Mol. Biotechnol. 19, 201-203.

Karibe, H. and Komatsu, S. (1997) Analysis of protein kinase involved in growth regulation of rice embryos and leaves. Biol. Pharm. Bull. 20, 1039-1043.

Kawasaki, T., Hayashida, N., Shinozaki, K. and Shimada, H. (1993) The gene encoding a calcium-dependent protein kinase located near the sbel gene encoding starch branching enzyme I is specifically expressed in developing rice seeds. Gene 129, 183-189.

Kim, K. N., Cheong, Y. H., Granta, J. J., Pandeya, G. K. and Luan, S. (2003) CIPK3, a calcium sensor-associated protein kinase that regulates abscisic acid and cold signal transduction in Arabidopsis. Plant Cell. 15, 411-423.

Komatsu, S., Li, W., Konishi, H., Yoshikawa, H., Konishi, H. and Yang, G. (2001) Characterization of a Ca2+-dependent protein kinase from rice root: differential response to cold and regulation by abscisic acid. Biol. Pharm. Bull. 24, 316-319.

Lee, S. S., Cho, H. S., Yoo, G. M., Ahn, J. W., Kim, H. H. and 
Pai, H. S. (2003) Interaction of NtCDPK1 calcium-dependent protein kinase with NtRpn3 regulatory subunit of the $26 \mathrm{~S}$ proteasome in Nicotiana tabacum. Plant J. 33, 825-840.

Levin, D. A. (1983) Polyploidy and novelty in flowering plants. Am. Nat. 122, 1-25.

Li, W. G. and Komatsu, S. (2000) Cold stress-induced calciumdependent protein kinase(s) in rice (Oryza sativa L.) seedling stem tissues. Theor. Appl. Genet. 101, 335-363.

Liu, B. and Wendel, J. F. (2003) Epigenetic phenomena and the evolution of plant allopolyploids. Mol. Phylogenet. Evol. 29, 365-379.

Martin, M. L. and Busconi, L. (2000) Membrane localization of a rice calcium-dependent protein kinase (CDPK) is mediated by myristoylation and palmitoylation. Plant J. 24, 429-435.

Madlung, A., Masuelli, R. W., Watson, B., Reynolds, S. H., Davison, J. and Comai, L. (2002) Remodeling of DNA methylation and phenotypic and transcriptional changes in synthetic Arabidopsis allotetraploids. Plant Physiol. 129, 733746.

Nordin, K., Heino P. and Palva, E. T. (1991) Separate signal pathways regulate the expression of a low-temperature-induced gene in Arabidopsis thaliana (L.) Heynh. Plant Mol. Biol. 16, 1061-1071.

Nordin, K., Vahala, T. and Palva, E. T. (1993) Differential expression of two related low-temperature induced genes in Arabidopsis thaliana L. Heynh. Plant Mol. Biol. 21, 641-653.

Osborn, T. C., Pires, J. C., Birchler, J. A., Auger, D. L., Chen, Z. J., Lee, H. S., Comai, L., Madlung, A., Doerge, R. W., Colot, V. and Martienssen, R. A. (2003) Understanding mechanisms of novel gene expression in polyploids. Trends Genet. 19, 141147.

Pires, J. C., Zhao, J., Schranz, M. E., Leon, E. J., Quijada, P. A., Lukens, L. and Osborn, T. C. (2004) Flowering time divergence and genomic rearrangements in resynthesized Brassica polyploids (Brassicaceae). Biol. J. Linn. Soc. 82, 675688.

Poovaiah, B. W. and Reddy, A. S. N. (1993) Calcium and signal transduction in plant. Crit. Rev. Plant Sci. 12, 185-211.

Qiao, C. Z., Wu, M. S., Dai, F. B., Cui, X. and Li, L. (1989) Studies on polyploid breeding of Isatis indigotica Fort. Acta. Bot. Sin. 31, 678-683.

Rabbani, M. A., Maruyama, K., Abe, H., Khan, M. A., Katsura, K., Ito, Y., Yoshiwara, K., Seki, M., Shinozaki, K. and Yamaguchi-Shinozaki, K. (2003) Monitoring expression profiles of rice genes under cold, drought, and high-salinity stresses and abscisic acid application using cDNA microarray and RNA gel-Blot analyses. Plant Physiol. 133, 1755-1767.

Ramsey, J. and Schemske, D. W. (2002) Neopolyploidy in flowering plants. Annu. Rev. Ecol. Syst. 33, 589-639.

Rechards, E. J. (1995) Preparation and analysis of DNA; in Short
Protocol in Molecular Biology, Ausubel, F. M., Brent, R., Kingston, R. E., Moore, D. D., Seidman, J. G., Smith, J. A. and Struhl, K. (eds.), pp. 36-38, John Wiley and Sons, New York, USA.

Romeis, T., Ludwig, A. A., Matin, R. and Jones, J. D. J. (2001) Calcium-dependent protein kinase plays an essential role in a plant defense response. EMBO J. 20, 5556-5567.

Rutschmann, F., Stalder, U., Piotrowski, M., Oecking, C. and Schaller, A. (2002) LeCPK1, a calcium-dependent protein kinase from tomato. Plasma membrane targeting and biochemical characterization. Plant Physiol. 129, 156-168.

Shapiro, M. B. and Senapathy, P. (1987) RNA splice junctions of different classes of eukaryotes; Sequece statistics and functional implications in gene expression. Nucleic. Acids. Res. 15, 71557174.

Shen, Y., Jia, W., Zhang, Y., Hu, Y., Wu, Q. and Lin Z. (2004) Improvement of drought tolerance in transgenic tobacco plants by a dehydrin-Like gene transfer. Agri. Sci. China. 3, 575-583.

Sopory, S. K. and Munshi, M. (1998) Protein kinases and phosphatases and their role in cellular signaling in plants. Crit. Rev. Plant Sci. 17, 245-318.

Urao, T., Katagiri, T., Mizoguchi, T., Yamaguchi-Shinozaki, K., Hayashida, N. and Shinozaki, K. (1994) Two genes that encode $\mathrm{Ca}+2$-dependent protein kinases are induced by drought and highsalt stresses in Arabidopsis thaliana. Mol. Gen. Genet. 244, 331-340.

Wendel, J. F. (2000) Genome evolution in polyploids. Plant Mor. Biol. 42, 225-249.

Yamaguchi-Shinozaki, K. and Shinozaki, K. (1994) A novel cisacting element in an Arabidopsis gene is involved in responsiveness to drought, low-temperature, or high-salt stress. Plant Cell. 6, 251-264.

Yamaguchi-Shinozaki, K., Koizumi, M., Urao, S. and Shinozaki, K. (1992) Molecular cloning and characterization of 9 cDNAs for genes that are responsive to desiccation in Arabidopsis thaliana: sequence analysis of one cDNA clone that encodes a putative transmembrane channel protein. Plant Cell Physiol. 33, 217-224.

Yamaguchi-Shinozaki, K. and Shinozaki, K. (1993) Characterization of the expression of a desiccation responsive rd29 gene of Arabidopsis thaliana and analysis of its promoter in transgenic plants. Mol. Gen. Genet. 236, 331-340.

Yang, G. and Komatsu, S. (2000) Involvement of calciumdependent protein kinase in rice lamina inclination caused by brassinolide. Plant Cell Physiol. 41, 1243-1250.

Zhao, J., Davis, L. C. and Verpoorte, R. (2005) Elicitor signal transduction leading to production of plant secondary metabolites. Biotechnol. Adv. 23, 283-333.

Zhu, J. K. (2002) Salt and drought stress signal transduction in plants. Annu. Rev. Plant Biol. 53, 247-273. 